\title{
Synthese und Eigenschaften von 1,2,4,3-Thiadiazaboretidinen. Kristallstruktur des 2,4-Di-tert-butyl-3-phenyl-1,2,4,3-thiadiazaboretidins
}

\author{
Carl D. Habben* a, Andreas Heine ${ }^{a}$, George M. Sheldrick ${ }^{a}$, Dietmar Stalke ${ }^{\text {a }}$, Michael Bühl ${ }^{b}$ und Paul von R. Schleyer $^{b}$
}

Institut für Anorganische Chemie der Universität Göttingen a,

Tammannstraße 4, D-3400 Göttingen

Institut für Organische Chemie der Universität Erlangen-Nürnberg ${ }^{b}$,

Henkestraße 42, D-8520 Erlangen

Eingegangen am 18. Mai 1990

Key Words: Sulfur diimides / 1,3-Cycloaddition, reductive / 1,2,4,3-Thiadiazaboretidines

Synthesis and Properties of 1,2,4,3-Thiadiazaboretidines. Crystal Structure of 2,4-Di-tert-butyl-3-phenyl-1,2,4,3thiadiazaboretidine

Reaction of sulfur diimides with alkyl(aryl)bis(methylthio)boranes leads to reductive 1,3-cycloaddition of the NSN sequence with formation of the 1,2,4,3-thiadiazaboretidines $2 \mathbf{a}-\mathbf{g}$. NMR $\left({ }^{1} \mathrm{H},{ }^{11} \mathrm{~B},{ }^{13} \mathrm{C},{ }^{15} \mathrm{~N},{ }^{29} \mathrm{Si}\right)$, mass spectra and the results of the $\mathrm{X}$ ray analysis of $2 f$ are reported and discussed. The ${ }^{11} \mathrm{~B}$ chemical shifts have been calculated for 3-phenyl-1,2,4,3-thiadiazaboretidine and some related model compounds by the IGLO method and correlate acceptably with the experimental values.

Wie kürzlich gezeigt ${ }^{11}$, reagiert 3,5-Dimethyl-1,2,4,3,5-trithiadiborolan mit Di-tert-butylschwefeldiimid zunächst zum 1,4,2,6,3,5-Dithiazadiborinan 1 a, das von weiterem Schwefeldiimid zum 1,2,4,3-Thiadiazaboretidin $\mathbf{2 a}$ abgebaut wird, Gl. (1).

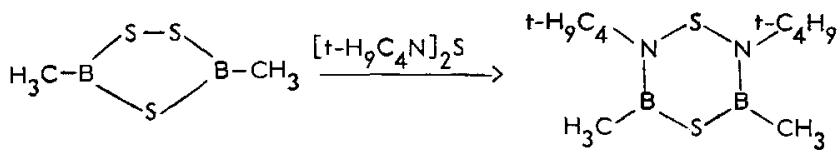

la

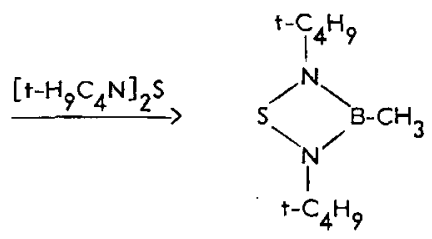

$2 a$

Da bisher nur 2a darstellbar war, sollte ein neuer Syntheseweg gefunden werden. Thiadiazaboretidine 2 sind insofern von Interesse, als sie den formalen Übergang zwischen den gut bekannten Diazadiboretidinen $(\mathbf{A})^{2-5)}$ und den wenig untersuchten Dithiadiazaetidinen $(\mathbf{C})^{6}$ bilden.<smiles>[R]B1N([R])BN1[R]</smiles>

A<smiles>[R]N1[R]N([R])S1</smiles>

8<smiles>[R]N1SN([R])S1</smiles>

c

\section{Ergebnisse und Diskussion}

Schwefeldiimide reagieren mit Alkyl(Aryl)bis(methylthio)boranen zu 1,2,4,3-Thiadiazaboretidinen 2.

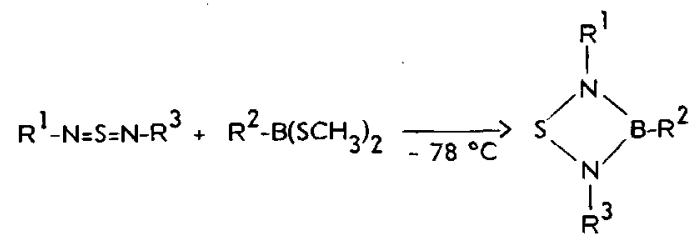

\begin{tabular}{c|cll} 
Verb. & $R^{1}$ & \multicolumn{1}{c}{$R^{2}$} & \multicolumn{1}{c}{$R^{3}$} \\
\hline 2a & $t-\mathrm{C}_{4} \mathrm{H}_{9}$ & $\mathrm{CH}_{3}$ & $t-\mathrm{C}_{4} \mathrm{H}_{9}$ \\
b & $t-\mathrm{C}_{4} \mathrm{H}_{9}$ & $\mathrm{CH}_{3}$ & $\mathrm{Si}\left(\mathrm{CH}_{3}\right)_{3}$ \\
c & $t-\mathrm{C}_{4} \mathrm{H}_{9}$ & $\mathrm{C}_{2} \mathrm{H}_{5}$ & $t-\mathrm{C}_{4} \mathrm{H}_{9}$ \\
d & $t-\mathrm{C}_{4} \mathrm{H}_{9}$ & $\mathrm{C}_{2} \mathrm{H}_{5}$ & $\mathrm{Si}\left(\mathrm{CH}_{3}\right)_{3}$ \\
e & $\mathrm{i}-\mathrm{C}_{3} \mathrm{H}_{7}$ & $\mathrm{C}_{6} \mathrm{H}_{5}$ & $\mathrm{Si}\left(\mathrm{CH}_{3}\right)_{3}$ \\
f & $t-\mathrm{C}_{4} \mathrm{H}_{9}$ & $\mathrm{C}_{6} \mathrm{H}_{5}$ & $\mathrm{t}-\mathrm{C}_{4} \mathrm{H}_{9}$ \\
g & $t-\mathrm{C}_{4} \mathrm{H}_{9}$ & $\mathrm{C}_{6} \mathrm{H}_{5}$ & $\mathrm{Si}\left(\mathrm{CH}_{3}\right)_{3}$
\end{tabular}

Die Umsetzung erfolgt unter Reduktion des DiimidSchwefel-Atoms und 1,3-Cycloaddition der DiaminosulfanEinheit an das Bor-Atom.

Die Reaktionen sind in der Kälte $\left(-78^{\circ} \mathrm{C}\right)$ durchzuführen, da bei Raumtemperatur oder unter Rückflußbedingungen und ohne Lösungsmittel nur Polykondensate entstehen, deren Bildung in der Kälte gemäß Gl. (2) als Nebenprodukt beobachtet wird. Die Reaktivität von (Alkylamino)bis(me- 
thylthio)boranen reicht nicht mehr zur Bildung von aminosubstituierten Thiadiazaboretidinen aus.

Tab. 1. Massenspektrometrische und NMR-Daten $\left({ }^{1} \mathrm{H},{ }^{11} \mathrm{~B},{ }^{13} \mathrm{C},{ }^{15} \mathrm{~N}\right.$, $\left.{ }^{29} \mathrm{Si}\right)$ der Verbindungen $2 \mathrm{a}-\mathrm{g}$. (c) $\mathrm{B}-\mathrm{C}$ nicht registrierbar

\begin{tabular}{|c|c|c|c|c|c|c|c|}
\hline Verb. & $\begin{array}{l}\text { MS } \mathrm{m} / \mathrm{z} \\
\mathrm{M}^{+} / \mathrm{Int} . \\
\text { [Bosispe }\end{array}$ & & $\begin{array}{c}{ }_{H} \\
\delta\end{array}$ & & $\begin{array}{c}{ }^{13} \mathrm{C} \\
\delta\end{array}$ & $\begin{array}{c}11_{B} \\
6\end{array}$ & $\begin{array}{c}29 \\
15 \mathrm{Si}(\mathrm{a}) \\
\mathrm{N}(\mathrm{b}) \\
\delta\end{array}$ \\
\hline \multirow[t]{2}{*}{$2 a$} & $200 / 26$ & $\mathrm{~B}-\mathrm{CH}_{3}$ & 0.37 & & 1.1 & 29.8 & $-271,7(b)$ \\
\hline & [88] & $\begin{array}{l}\mathrm{C}\left(\mathrm{CH}_{3}\right)_{3} \\
\mathrm{C}\left(\mathrm{CH}_{3}\right)_{3}\end{array}$ & 1.24 & & 30.14 & 52.62 & \\
\hline \multirow[t]{4}{*}{ b } & $216 / 41$ & $\mathrm{Si}\left(\mathrm{CH}_{3}\right)_{3}$ & 0.16 & {$[9 \mathrm{H}]$} & 0.09 & 30.5 & $5.9(0)$ \\
\hline & [57] & $\mathrm{B}-\mathrm{CH}_{3}$ & 0.29 & {$[3 \mathrm{H}]$} & 1.2 & & \\
\hline & & $\mathrm{C}\left(\mathrm{CH}_{3}\right)_{3}$ & 1.24 & (s) $[9 \mathrm{H}]$ & 30.35 & & \\
\hline & & $\mathrm{C}\left(\mathrm{CH}_{3}\right)_{3}$ & & & 52.66 & & \\
\hline \multirow[t]{4}{*}{ c } & $214 / 25$ & $\mathrm{CH}_{2}$ & 0.82 & {$[2 \mathrm{H}]$} & 9.1 & 30.8 & \\
\hline & {$[57]$} & $\mathrm{CH}_{3}$ & 1.01 & (t) $[3 \mathrm{H}]$ & 9.61 & & \\
\hline & & $\mathrm{C}\left(\mathrm{CH}_{3}\right)_{3}$ & 1.25 & (s) $[18 \mathrm{H}]$ & 30.16 & & \\
\hline & & $\mathrm{C}\left(\mathrm{CH}_{3}\right)_{3}$ & & & 52.67 & & \\
\hline \multirow[t]{5}{*}{ d } & $230 / 26$ & $\mathrm{Si}\left(\mathrm{CH}_{3}\right)_{3}$ & 0.18 & (s) $[9 \mathrm{H}]$ & 0.15 & 31.6 & $5.8(a)$ \\
\hline & & $\mathrm{CH}_{2}$ & 0.74 & (q) $[2 \mathrm{H}]$ & 8.9 & & \\
\hline & & $\mathrm{CH}_{3}$ & 0.99 & $(\uparrow) \quad[3 \mathrm{H}]$ & 9.67 & & $-263.4(b)$ \\
\hline & & $\mathrm{C}\left(\mathrm{CH}_{3}\right)_{3}$ & 1.26 & {$[9 \mathrm{H}]$} & 30.41 & & -306.7 \\
\hline & & $\underline{\mathrm{C}}\left(\mathrm{CH}_{3}\right)_{3}$ & & & 52.73 & & \\
\hline \multirow[t]{4}{*}{ e } & $264 / 17$ & $\mathrm{Si}\left(\mathrm{CH}_{3}\right)_{3}$ & 0.28 & (s) $[9 \mathrm{H}]$ & 0.96 & 32.8 & \\
\hline & [73] & $\mathrm{CH}_{3}$ & 1.28 & (d) [6H] & 32.43 & & \\
\hline & & $\mathrm{CH}$ & 4.06 & (sp) $[\mathrm{lH}]$ & 51.34 & & \\
\hline & & $\mathrm{C}_{6} \mathrm{H}_{5} 7.33-$ & -7.61 & (br) $[5 \mathrm{H}]$ & $\begin{array}{c}127.43 \\
132.96 \\
133.94 \\
(c)\end{array}$ & & \\
\hline \multirow[t]{2}{*}{ f } & $262 / 41$ & $\mathrm{C}\left(\mathrm{CH}_{3}\right)_{3}$ & 1.16 & (s) $[18 \mathrm{H}]$ & $\begin{array}{l}30.30 \\
53.34\end{array}$ & 28.5 & \\
\hline & {$[57]$} & $\frac{\mathrm{C}\left(\mathrm{CH}_{3}\right)_{3}}{\mathrm{C}_{6} \mathrm{H}_{5}}$ & $\begin{array}{l}7.26- \\
7.41\end{array}$ & (br) $[5 \mathrm{H}]$ & $\begin{array}{c}127.21 \\
128.32 \\
132.77 \\
\text { (c) }\end{array}$ & & -268.2 (b) \\
\hline \multirow[t]{5}{*}{$\mathbf{g}$} & $278 / 53$ & $\mathrm{Si}\left(\mathrm{CH}_{3}\right)_{3}$ & 0.16 & (s) $[9 \mathrm{H}]$ & 0.20 & 28.7 & $7.2(\mathrm{o})$ \\
\hline & [57] & $\mathrm{C}\left(\mathrm{CH}_{3}\right)_{3}$ & 1.34 & (s) $[9 \mathrm{H}]$ & 30.70 & & \\
\hline & & $\mathrm{C}\left(\mathrm{CH}_{3}\right)_{3}$ & & & 53.33 & & $-259.0(b)$ \\
\hline & & $\mathrm{C}_{6} \mathrm{H}_{5}$ & & & $\begin{array}{l}127.39 \\
128.72 \\
132.72\end{array}$ & & -301.9 \\
\hline & & $B-C$ & & & 137.2 & & \\
\hline
\end{tabular}

\section{Spektren}

Spektroskopische Daten sind in Tab. 1 zusammengefaßt. Die EI-Massenspektren der Thiadiazaboretidine zeigen Molekülpeaks mit nur mittleren relativen Intensitäten $\left(17-53 \%\right.$ ). Als Basispeak (für $\mathrm{R}^{1,3}=t-\mathrm{C}_{4} \mathrm{H}_{9}$ ) tritt übereinstimmend (Ausnahme 2a, 90\%) ein Fragment $m / z=57$ auf, welches dem Ion $\left[\mathrm{C}_{4} \mathrm{H}_{9}\right]^{+}$entspricht. Für $\mathrm{R}^{1}=i-\mathrm{C}_{3} \mathrm{H}_{7}$ in 2e wird ein entsprechendes Fragment bei $m / z=44$ (für $\left[\mathrm{C}_{4} \mathrm{H}_{8}\right]^{+}$) mit einer relativen Intensität von $76 \%$ gefunden. Der Basispeak liegt hier bei $m / z=73$ für $\left[\mathrm{Si}\left(\mathrm{CH}_{3}\right)_{3}\right]^{+}$, einer Massenzahl, bei der in den Spektren der Trimethylsilyl-substituierten Derivate $\mathbf{2} \mathbf{b}, \mathbf{d}$ und $\mathbf{g}$ intensive Signale registriert werden (rel. Int. [\%]: 94, 56, 86). Durch MethylgruppenAbstraktion aus $M$ entstandene Fragmente werden bei $2 \mathbf{c}-\mathbf{f}$ mit geringer $(10-16 \%)$, bei $\mathbf{a}, \mathbf{g}$ und $\mathbf{b}$ mit höherer $(20,25$ bzw. $36 \%)$ relativer Intensität registriert. In jedem
Fall liegt der für $[\mathrm{M}]^{+}$geltende Wert um $5(2 \mathrm{~b})$ bis $28 \%$ (2g) höher.

Die ${ }^{1} \mathrm{H}$ - und ${ }^{13} \mathrm{C}$-NMR-Spektren der Thiadiazaboretidine zeigen die erwarteten Signale in Lage und Intensität.

Die für 2 gemessenen ${ }^{11} \mathrm{~B}-\mathrm{NMR}$-Verschiebungen liegen mit $\delta=28.5-32.8 \mathrm{im}$ Bereich der für cyclische Diaminoorganylborane und Diorganyldiazaboretidine ${ }^{7}$ gefundenen Werte. Der für 2a in Lit. ${ }^{1)}$ angegebene Wert von $\delta=$ 35.5 kann nicht bestätigt werden. Bei vergleichbaren Thiadiazaboretidinen wird sowohl der Substituentenwechsel am Bor- $\left(\mathrm{CH}_{3} \rightarrow \mathrm{C}_{2} \mathrm{H}_{5}\right)$ als auch am Stickstoff- $\left[t-\mathrm{C}_{4} \mathrm{H}_{9} \rightarrow\right.$ $\mathrm{Si}\left(\mathrm{CH}_{3}\right)_{3}$ ]-Atom jeweils von einer Tieffeldverschiebung um ca. $1 \mathrm{ppm}$ begleitet, nicht jedoch bei $\mathrm{R}^{2}=\mathrm{C}_{6} \mathrm{H}_{5}$ und Ersatz von $t-\mathrm{C}_{4} \mathrm{H}_{9}$ durch $\mathrm{Si}\left(\mathrm{CH}_{3}\right)_{3}$. Die unterschiedliche elektronische Wirkung der $\mathrm{N}$-ständigen $-\mathrm{C}\left(\mathrm{CH}_{3}\right)_{3-}$ bzw. $-\mathrm{CH}\left(\mathrm{CH}_{3}\right)_{2}$-Gruppen auf das Ringsystem spiegelt sich dagegen in einer Tieffeldverschiebung $(\mathbf{2} \mathbf{f}, \mathbf{g} \rightarrow \mathbf{e})$ um $4 \mathrm{ppm}$ wider.

An den ${ }^{15}$ N-NMR-Daten ist der Substituentenwechsel am Ringgerüst ebenfalls zu verfolgen. Während beide StickstoffAtome in $\mathbf{2 f}$ nicht unterscheidbar sind, liefert $\mathbf{2 g}$ zwei Signale. Der Ersatz einer tert-Butyl- durch eine Trimethylsilylgruppe führt bei der in $\mathbf{2 f}$ und $\mathbf{2} \mathrm{g}$ angegebenen Zuordnung $\mathrm{zu}$ einer leicht erhöhten Abschirmung für $\mathrm{N}-2$ $\left(N-\mathrm{C}_{4} \mathrm{H}_{9}\right)$ in $\mathbf{2 g}$ im Vergleich mit 2f. Dieser Effekt wird auch bei $2 \mathrm{~d}$ beobachtet.

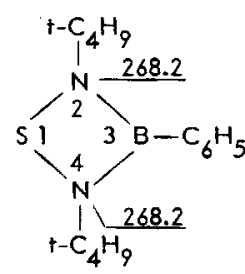

$2 f$

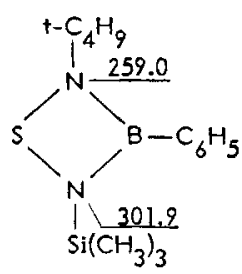

$2 g$
Mit $\delta=5.8-7.2$ liegen die ${ }^{29} \mathrm{Si}-\mathrm{NMR}$-Werte im Vergleich $z \mathfrak{u}$ denen Trimethylsilyl-substituierter Dithiazadiborinane ${ }^{8)}$ bei höherem Feld $\left(\mathrm{BCH}_{3}\right.$ 14.3) bzw. im gleichen Bereich $\left[\mathrm{BN}\left(\mathrm{C}_{2} \mathrm{H}_{5}\right)_{2} 6.7\right]$.

Die theoretische Berechnung der ${ }^{11} \mathrm{~B}$ chemischen Verschiebung einer Modellverbindung $\left(R^{1}=R^{3}=H, R^{2}=\right.$ $\mathrm{C}_{6} \mathrm{H}_{5}$ ) mit Hilfe der IGLO-Methode (Individual Gauge for Localized Orbitals) ${ }^{9)}$ liefert $\delta=33.9$ (IGLO Double ZetaBasis, exptl. Geometrie). Dieser Wert liegt am entschirmten Ende des experimentellen Bereichs.

Tabelle 2 zeigt die Ergebnisse analoger IGLO-Rechnungen für Modellverbindungen kürzlich synthetisierter und röntgenographisch charakterisierter Aminoorganylborane $^{10)}$. Die Übereinstimmung mit den experimentellen chemischen Verschiebungen ist zufriedenstellend; die maximale Abweichung beträgt ca. 5 ppm. Der Einfluß der unterschiedlichen Substituenten sowie der Effekt größerer Basissätze wird jedoch noch an kleineren Modellsystemen zu testen sein.

Insgesamt scheint für diese „klassischen“ dreifach koordinierten Borverbindungen die Zugrundelegung von Rönt- 
genstrukturen in den Rechnungen befriedigende Ergebnisse zu liefern; für die polyedrischen Borane dagegen sind Abinitio-Geometrien (auf hohem Niveau optimiert) vorzuziehen $^{9 \mathrm{~d})}$.

Tab. 2. Theoretische (IGLO) ${ }^{\text {a) }}$ und experimentelle "B-chemische Verschiebungen von Aminoorganylboranen

\begin{tabular}{llll}
\hline Verbindung & IGLO & Expt. & Verbindung \\
& $\delta{ }^{11} \mathrm{~B}$ & $\delta^{11} \mathrm{~B}$ & \\
\hline
\end{tabular}

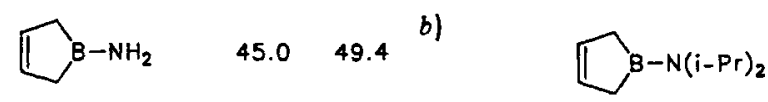<smiles>NB1C2C=CC1C=C2</smiles><smiles>[13CH3][AsH2]</smiles><smiles>[PH2+]C1C2C=CC([PbH2])C1C=C2</smiles><smiles>NB1CCB(N)B1N</smiles>
49.2
$\times 62.8$
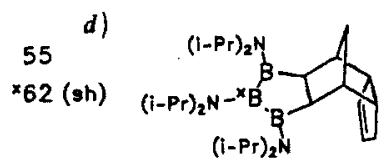<smiles>c1ccc(B2NSN2)cc1</smiles>

33.9

28.5 e)

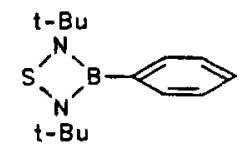

a) IGLO Basis Double Zeta (DZ), Geometrien aus Röntgenstrukturanalysen, sperrige Reste durch Wasserstoff $(\mathrm{NH}=1.0, \mathrm{CH}=$ $1.08 \AA$ ) ersetzt. - b) Lit. ${ }^{10 a)}$. - ${ }^{\text {c) }}$ Lit. $^{10 b)}$. - d) Lit. ${ }^{10 c)}$. - e) Diese Arbeit.

\section{Diskussion der Struktur von $2 \mathrm{f}$}

In Abb. 1 ist die Molekülstruktur des planaren Thiadiazaboretidins $2 \mathrm{f}$ wiedergegeben. Der zu 143.9(2) pm bestimmte BN-Bindungsabstand liegt im Bereich der für Borazine $^{11)}(139-144 \mathrm{pm})$ und 1,3,2,4-Diazadiboretidine $A\left(R^{1}\right.$

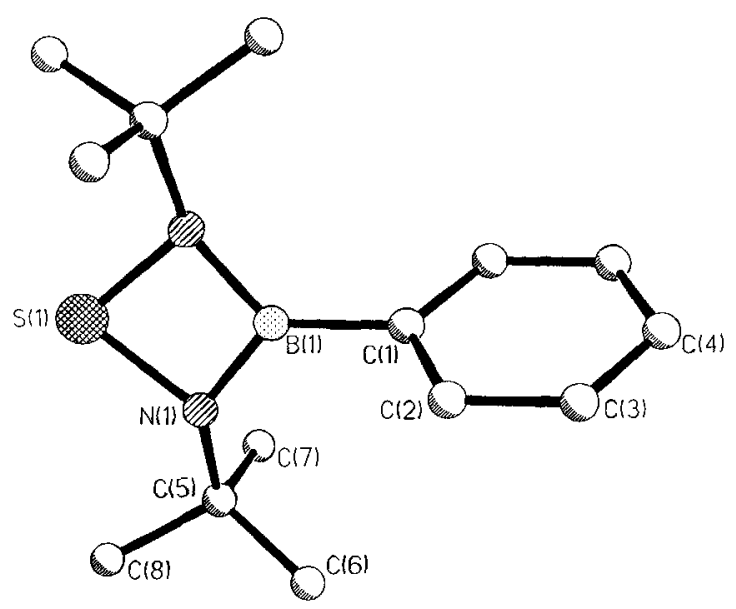

Abb. 1. Molekülstruktur von Verbindung $2 \mathbf{f}$
$\left.=t-\mathrm{C}_{4} \mathrm{H}_{9}, \mathrm{R}^{2}=\mathrm{C}_{6} \mathrm{~F}_{5}: 142.6-143.6\right)^{12)}$ gefundenen Werte. Mit 173.5 pm entspricht der NS-Bindungsabstand einer formalen Einfachbindung, wie sie auch in 1,4,2,6,3,5-Dithiazadiborinanen ${ }^{13)}$ vorliegt (im Mittel 172 pm). Für acyclische, symmetrisch substituierte Schwefeldiimide wurden dagegen SN-Abstände von $151-158 \mathrm{pm}^{14)}$ gefunden. Der intramolekulare nichtbindende BS-Abstand beträgt $228.9 \mathrm{pm}$. Der dem N'BN-Winkel in 1,3-Di-tert-butyl-2,4-bis(pentafluorphenyl)-1,3,2,4-diazadiboretidin $\left(95.7^{\circ}\right)$ in $\mathbf{2 f}$ entsprechende Winkel $\mathrm{N}(1) \mathrm{B}(1) \mathrm{N}(1 \mathrm{~A})$ liegt geringfügig aufgeweitet vor $\left(98.5^{\circ}\right)$, der gegenüberliegende $\mathrm{N}(1) \mathrm{S}(1) \mathrm{N}(1 \mathrm{~A})$-Winkel ist mit $77.8^{\circ}$ nicht so spitz wie der BSB-Winkel in 2,4-Bis(diisopropylamino)-3-phenyl-1,3,2,4-thiazadiboretidin $\left(70^{\circ}\right)^{15)}$. Der Torsionswinkel in $\mathbf{2 f}$ Thiadiazaboretidin/Phenyl wurde $\mathbf{z u}$ $65^{\circ}$ bestimmt.

Für die Förderung dieser Arbeit danken wir dem Fonds der Chemischen Industrie (Mittel über Professor A. Meller) und dem Leibniz-Programm der Deutschen Forschungsgemeinschaft (G.M.S.).

\section{Experimenteller Teil}

C,H-Bestimmungen: Mikroanalytisches Laboratorium Beller, Göttingen. - NMR (Solvens/Standard): ${ }^{1} \mathrm{H}\left(\mathrm{CDCl}_{3} / \mathrm{TMS}\right.$ int.): Bruker WP $80 \mathrm{SY},{ }^{11} \mathrm{~B}\left[\mathrm{CDCl}_{3} / \mathrm{BF}_{3}-\mathrm{O}\left(\mathrm{C}_{2} \mathrm{H}_{5}\right)_{2}\right.$ ext. $],{ }^{13} \mathrm{C},{ }^{29} \mathrm{Si}$ ( $\mathrm{CDCl}_{3} / \mathrm{TMS}$ int.), ${ }^{15} \mathrm{~N}\left(\mathrm{CDCl}_{3} / \mathrm{H}_{3} \mathrm{CNO}_{2}\right.$ ext.): Bruker AM 250 . MS: $70 \mathrm{eV}$, Varian MAT-CH 5-Spektrometer. Molekülpeaks sind durch Feldionisation gesichert. - Ausgangsverbindungen: Di-tertbutyl- ${ }^{16)}$, tert-Butyl(isopropyltrimethylsilyl)- ${ }^{17}$ schwefeldiimid und Alkyl(Phenyl)bis(methylthio)borane ${ }^{18)}$ wurden nach Literaturangaben dargestellt. - Alle Reaktionen wurden unter Ausschluß von Feuchtigkeit unter $\mathrm{N}_{2}$ und in getrockneten Lösungsmitteln durchgeführt.

2,4-Di-tert-butyl-3-methyl- (2a), 2-tert-Butyl-3-methyl-4-(trimethylsilyl)- (2b), 2,4-Di-tert-butyl-3-ethyl- (2c), 2-tert-Butyl-3-ethyl-4(trimethylsilyl)- (2d), 2-Isopropyl-3-phenyl-4-(trimethylsilyl)-(2e), 2,4-Di-tert-butyl-3-phenyl- (2f) und 2-tert-Butyl-3-phenyl-4-(trimethylsilyl)-(2g) -1,2,4,3-thiadiazaboretidin: In die auf $-78^{\circ} \mathrm{C}$ gekühlte Lösung von $10 \mathrm{mmol}$ des jeweiligen Schwefeldiimids $[1.74 \mathrm{~g}$

Tab. 3. Präparative und analytische Daten der Verbindungen $\mathbf{2 a}-\mathbf{g}$

\begin{tabular}{|c|c|c|c|c|c|c|}
\hline \multirow[t]{2}{*}{ Verb. } & \multirow[t]{2}{*}{$\begin{array}{c}\text { Ausb. } \\
\mathrm{g} / \%\end{array}$} & \multirow[t]{2}{*}{$\begin{array}{l}\text { Sdp. } \\
{\left[{ }^{\circ} \mathrm{C}\right]}\end{array}$} & \multirow[t]{2}{*}{$\underset{\left[{ }^{\circ} \mathrm{C}\right]}{\text { Schmp. }}$} & \multirow[t]{2}{*}{$\begin{array}{l}\text { Summenformel } \\
\text { Molmasse } \\
\text { Ber. Gef. (MS) }\end{array}$} & \multicolumn{2}{|c|}{$\begin{array}{c}\text { Analyse } \\
\text { Ber. } \\
\text { Gef. }\end{array}$} \\
\hline & & & & & & $\mathrm{H}$ \\
\hline $2 \mathbf{a}$ & $1.2 / 61$ & 28 & & $\begin{array}{l}\mathrm{C}_{9} \mathrm{H}_{21} \mathrm{BN}_{2} \mathrm{~S} \\
200.2200\end{array}$ & $\begin{array}{l}54.01 \\
54.12\end{array}$ & $\begin{array}{l}10.58 \\
10.54\end{array}$ \\
\hline b & $0.9 / 42$ & 35 & & $\begin{array}{l}\mathrm{C}_{8} \mathrm{H}_{21} \mathrm{BN}_{2} \mathrm{SSi} \\
216.2216\end{array}$ & $\begin{array}{l}44.44 \\
43.66\end{array}$ & $\begin{array}{l}9.79 \\
9.57\end{array}$ \\
\hline c & $1.2 / 56$ & 52 & & $\begin{array}{l}\mathrm{C}_{10} \mathrm{H}_{23} \mathrm{BN}_{2} \mathrm{~S} \\
214.2214\end{array}$ & $\begin{array}{l}56.08 \\
55.70\end{array}$ & $\begin{array}{l}10.82 \\
10.65\end{array}$ \\
\hline d & $1.2 / 52$ & 59 & & $\begin{array}{l}\mathrm{C}_{9} \mathrm{H}_{23} \mathrm{BN}_{2} \mathrm{SSi} \\
230.2230\end{array}$ & $\begin{array}{l}46.95 \\
46.67\end{array}$ & $\begin{array}{l}10.07 \\
10.04\end{array}$ \\
\hline e & $1.0 / 38$ & 85 & & $\begin{array}{l}\mathrm{C}_{12} \mathrm{H}_{21} \mathrm{BN}_{2} \mathrm{SSi} \\
264.3264\end{array}$ & $\begin{array}{l}54.54 \\
54.55\end{array}$ & $\begin{array}{l}8.01 \\
8.66\end{array}$ \\
\hline f & $1.7 / 65$ & $80^{\text {a) }}$ & 116 & $\begin{array}{l}\mathrm{C}_{14} \mathrm{H}_{23} \mathrm{BN}_{2} \mathrm{~S} \\
262.2262\end{array}$ & $\begin{array}{l}64.12 \\
63.71\end{array}$ & $\begin{array}{l}8.84 \\
9.20\end{array}$ \\
\hline g & $2.0 / 72$ & $80^{\text {a) }}$ & 89 & $\begin{array}{l}\mathrm{C}_{13} \mathrm{H}_{23} \mathrm{BN}_{2} \mathrm{SSi} \\
278.3278\end{array}$ & $\begin{array}{l}56.11 \\
55.84\end{array}$ & $\begin{array}{l}8.33 \\
8.46\end{array}$ \\
\hline
\end{tabular}

\footnotetext{
a) Sublimationstemp. $\left[{ }^{\circ} \mathrm{C}\right]$ bei $10^{-2}$ mbar.
} 


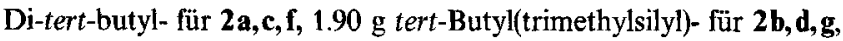
$1.76 \mathrm{~g}$ Isopropyl(trimethylsilyl)- für 2e] in $200 \mathrm{ml}$ Dichlormethan wurden langsam unter Rühren $10 \mathrm{mmol}$ Alkyl(Phenyl)bis(methylthio)boran (1.20 g Methyl- für 2a, b, $1.34 \mathrm{~g}$ Ethyl- für 2c, d, Phenylfür $2 \mathbf{e}-\mathbf{g}$ ), gelöst in $50 \mathrm{ml}$ Dichlormethan, getropft. Es folgten 12 stdg. Rühren bei $-78^{\circ} \mathrm{C}$, Auftauen auf Raumtemp., Abtrennung des Lösungsmittels und Produktdestillation bzw. -sublimation im Ölpumpenvakuum. Einkristalle von 2f wurden durch Umkristallisieren des Sublimates aus $n$-Hexan erhalten.

Tab. 4. Daten zur Kristallstrukturanalyse der Verbindung $2 \mathbf{f}$

Formel $\mathrm{C}_{14} \mathrm{H}_{23} \mathrm{BN}_{2} \mathrm{~S}$, Molmasse 262.23, Kristallgröße $0.5 \times 0.6 \times$ $0.4 \mathrm{~mm}$

$a=1374.2(8), b=1038.2(6), c=1131.6(6)$;

$\beta=110.82(5)^{\circ}, V=1.509 \mathrm{~nm}^{3}, D_{\text {Calc. }}=1.154$, Raumgruppe $C 2 / c$, $Z=4 \mu\left(\right.$ Mo $-K_{\alpha}$, Graphitmonochromator $)=0.38 \mathrm{~mm}^{-1}, \lambda=$ $71.069 \mathrm{pm}, T=103 \mathrm{~K} ; F(000)=1136$

STOE-Siemens-AED-Vierkreisdiffraktometer, Datensatz: Ausgewertet mit Profilfitting nach einem erlernten Profil ${ }^{20)}$

Anzahl der gemessenen Reflexe 1564, unabhängige Reflexe $1326\left(8^{\circ}\right.$ $\left.\leqslant 2 \Theta \leqslant 50^{\circ}\right)$, davon beobachtet $1193\left[F_{\mathrm{o}} \geqslant 3 \sigma\left(F_{\mathrm{o}}\right)\right]$

Lösung mit direkten Methoden, Strukturverfeinerung nach der Block-Kaskaden-Methode und Darstellung: SHELXTL-Programmsystem ${ }^{21)}$

$R=0.035, R_{w}=0.041, w^{-1}=\sigma^{2}(F)+0.0002 \cdot F^{2}$, max. Restelektronendichte $3.4 \times 10^{2} \mathrm{enm}^{-3}$, minimale Restelektronendichte $-2.8 \times 10^{2} \mathrm{enm}^{-3}$

Tab. 5. Atomkoordinaten $\left(\times 10^{4}\right)$ und äquivalente isotrope Auslenkungsparameter $\left(\mathrm{pm}^{2} \times 10^{-1}\right)$ für $\mathbf{2 f}$. Aquivalente isotrope $U$ berechnet als ein Drittel der Spur des orthogonalen $U_{i j}$ Tensors

\begin{tabular}{lccll}
\hline & $\mathrm{x}$ & $\mathrm{y}$ & $\mathrm{z}$ & $\mathrm{U}(\mathrm{eq})$ \\
\hline $\mathrm{S}(1)$ & 0 & $577(1)$ & 2500 & $18(1)$ \\
$\mathrm{N}(1)$ & $539(1)$ & $-723(1)$ & $1989(1)$ & $16(1)$ \\
$\mathrm{B}(1)$ & 0 & $-1628(2)$ & 2500 & $15(1)$ \\
$\mathrm{C}(1)$ & 0 & $-3130(2)$ & 2500 & $15(1)$ \\
$\mathrm{C}(2)$ & $-404(1)$ & $-3832(2)$ & $1368(2)$ & $20(1)$ \\
$C(3)$ & $-412(1)$ & $-5168(2)$ & $1370(2)$ & $26(1)$ \\
$C(4)$ & 0 & $-5835(2)$ & 2500 & $29(1)$ \\
$C(5)$ & $1580(1)$ & $-629(1)$ & $1895(1)$ & $18(1)$ \\
$C(6)$ & $1718(1)$ & $-1798(2)$ & $1149(2)$ & $24(1)$ \\
$C(7)$ & $2418(1)$ & $-617(2)$ & $3224(2)$ & $24(1)$ \\
$C(8)$ & $1626(1)$ & $611(1)$ & $1197(2)$ & $23(1)$ \\
\hline
\end{tabular}

Tab. 6. Bindungsabstände $[\mathrm{pm}]$ und Bindungswinkel $\left[{ }^{\circ}\right]$ in $\mathbf{2 f}$

\begin{tabular}{llll}
\hline$S(1)-N(1)$ & $173.5(1)$ & $S(1)-N(1 A)$ & $173.5(1)$ \\
$N(1)-B(1)$ & $143.9(2)$ & $N(1)-C(5)$ & $147.6(2)$ \\
$B(1)-C(1)$ & $155.9(3)$ & $B(1)-N(1 A)$ & $143.9(2)$ \\
$C(1)-C(2)$ & $140.6(2)$ & $C(1)-C(2 A)$ & $140.6(2)$ \\
$C(2)-C(3)$ & $138.7(2)$ & $C(3)-C(4)$ & $138.6(2)$ \\
$C(4)-C(3 A)$ & $138.6(2)$ & $C(5)-C(6)$ & $152.9(2)$ \\
$C(5)-C(7)$ & $153.5(2)$ & $C(5)-C(8)$ & $152.3(2)$
\end{tabular}

\begin{tabular}{lrlr}
$N(1)-S(1)-N(1 A)$ & $77.8(1)$ & $S(1)-N(1)-B(1)$ & $91.9(1)$ \\
$S(1)-N(1)-C(5)$ & $120.8(1)$ & $B(1)-N(1)-C(5)$ & $136.3(1)$ \\
$N(1)-B(1)-C(1)$ & $130.8(1)$ & $N(1)-B(1)-N(1 A)$ & $98.5(2)$ \\
$C(1)-B(1)-N(1 A)$ & $130.8(1)$ & $B(1)-C(1)-C(2)$ & $121.2(1)$ \\
$B(1)-C(1)-C(2 A)$ & $121.2(1)$ & $C(2)-C(1)-C(2 A)$ & $117.5(2)$ \\
$C(1)-C(2)-C(3)$ & $121.2(1)$ & $C(2)-C(3)-C(4)$ & $120.0(2)$ \\
$C(3)-C(4)-C(3 A)$ & $120.0(2)$ & $N(1)-C(5)-C(6)$ & $107.5(1)$ \\
$N(1)-C(5)-C(7)$ & $109.8(1)$ & $C(6)-C(5)-C(7)$ & $110.5(1)$ \\
$N(1)-C(5)-C(8)$ & $108.3(1)$ & $C(6)-C(5)-C(8)$ & $110.4(1)$ \\
$C(7)-C(5)-C(8)$ & $110.3(1)$ & & \\
\hline
\end{tabular}

Kristallstrukturanalyse von $\mathbf{2} \mathbf{f}^{19 \%}$ : Die Struktur wurde mit Hilfe direkter Methoden ${ }^{21)}$ und anschließender Differenz-Fourier-Syn- thesen gelöst und nach dem Kleinste-Quadrate-Verfahren verfeinert. Alle Nichtwasserstoff-Atome wurden anisotrop verfeinert. Die Wasserstoff-Atome wurden geometrisch ideal positioniert und nach dem „Reitermodell“" in die Verfeinerung einbezogen. Die Wasserstoff-Atome wurden isotrop und ihre Auslenkungsparameter in äquivalenten Gruppen frei verfeinert. 84 Parameter wurden verfeinert, das Gewichtsschema war $w^{-1}=\sigma^{2}(F)+0.0002 F^{2}$.

Kristalldaten, Atomkoordinaten sowie ausgewählte Bindungsabstände und -winkel sind in den Tabellen $4-6$ zusammengestellt.

\section{CAS-Registry-Nummern}

$\mathbf{2}\left(\mathbf{R}^{1}=\mathrm{R}^{3}=\mathrm{H}, \mathrm{R}^{2}=\mathrm{C}_{6} \mathrm{H}_{5}\right): 128685-57-4 / \mathbf{2} \mathbf{a}: 122345-47-5 / \mathbf{2} \mathbf{b}$ : 128685-52-9 / 2c: 128685-53-0 / 2d: 128685-54-1 / 2e: 128685 55-2 / 2f: $128685-56-3 / 2 \mathrm{~g}:$ 128708-31-6/t- $\mathrm{C}_{4} \mathrm{H}_{9} \mathrm{~N}=\mathbf{S}=\mathrm{NC}_{4} \mathrm{H}_{9}-t$ : 2056-74-8 $/ t-\mathrm{C}_{4} \mathrm{H}_{9} \mathrm{~N}=\mathrm{S}=\mathrm{NSi}\left(\mathrm{CH}_{3}\right)_{3}: 55712-30-6 / i-\mathrm{C}_{3} \mathrm{H}_{7} \mathrm{~N}=\mathrm{S}=$ $\mathrm{NSi}\left(\mathrm{CH}_{3}\right)_{3}: 128685-51-8 / \mathrm{CH}_{3} \mathrm{~B}\left(\mathrm{SCH}_{3}\right)_{2}: 19163-08-7 / \mathrm{C}_{2} \mathrm{H}_{5-}$ $\mathrm{B}\left(\mathrm{SCH}_{3}\right)_{2}: 67340-63-0 / \mathrm{C}_{6} \mathrm{H}_{5} \mathrm{~B}\left(\mathrm{SCH}_{3}\right)_{2}: 17381-61-2$

1) C. Habben, A. Meller, S. Pusch, Z. Naturforsch., Teil B, 43 (1989) 959.

2) P. Schreyer, P. Paetzold, R. Boese, Chem. Ber. 121 (1988) 195.

3) W. Maringgele, The Chemistry of Inorganic Homo- and Heterocycles, Bd. 1, Academic Press Inc., London 1987.

4) T. Franz, E. Hanecker, H. Nöth, W. Stöcker, W. Storch, G. Winter, Chem. Ber. 119 (1986) 900.

${ }^{5)}$ P. Geymayer, E. G. Rochow, U. Wannagat, Angew.Chem. 76 (1964) 499; Angew. Chem. Int. Ed. Engl. 3 (1964) 633.

${ }^{6}$ H. W. Roesky, Adv. Inorg. Chem. 22 (1979) 240.

${ }^{7)}$ H. Nöth, B. Wrackmeycr, Nuclear Magnetic Resonance Spectroscopy of Boron Compounds, Springer Verlag, Berlin, Heidelberg, New York 1978.

8) C. Habben, A. Meller, Z. Naturforsch., Teil B, 39 (1984) 1022.

9) 9a) W. Kutzelnigg, Isr. J. Chem. 19 (1980) 193. - ${ }^{\text {b) }}$ M. Schindler, W. Kutzelnigg, J. Chem. Phys. 76 (1982) 1919. - 9c) Ubersicht: W. Kutzelnigg, M. Schindler, U. Fleischer, NMR, Basic Principles and Progress, Springer Verlag, Berlin 1990, im Druck. -

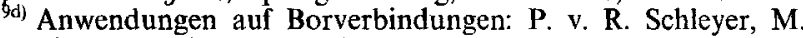
Bühl, U. Fleischer, W. Koch, Inorg. Chem. 29 (1990) 153; P. v. R. Schleyer, M. Bühl, Angew. Chem. 102 (1990) 320; Angew. Chem. Int. Ed. Engl. 29 (1990) 304; M. Bühl, P. v. R. Schleyer in Electron Deficient Boron and Carbon Clusters (G. A. Olah, K. Wade, R. E. Williams, Hrsg.), Wiley, New York 1990, im Druck.

10) 10a) D. Bromm, D. Stalke, A. Heine, A. Meller, G. M. Sheldrick, J. Organomet. Chem., im Druck. - ${ }^{10 \mathrm{~b})} \mathrm{W}$. Maringgele, D. Stalke, A. Heine, A. Meller, G. M. Sheldrick, Chem. Ber. 123 (1990) 489 . - ${ }^{10 \mathrm{c})}$ A. Meller, D. Bromm, W. Maringgele, A. Heine, D. Stalke, G. M. Sheldrick, Chem. Ber. 123 (1990) 293.

11) Gmelin, Handbuch der Anorganischen Chemie, Bd. 51, Borverbindungen, Teil 17, Springer Verlag, Berlin, Heidelberg, New York 1978

12) P. Paetzold, A. Richter, T. Thijssen, S. Würtenberg, Chem. Ber. 112 (1979) 3811.

13) C. Habben, A. Meller, M. Noltemeyer, G. M. Sheldrick, J. Organomet. Chem. 288 (1985) 1.

14) W. Isenberg, Dissertation, Universität Göttingen, 1984

15) C. Habben, M. Noltemeyer, Z. Naturforsch., Teil B, 44 (1989) 853.

16) D. H. Clemens, A. J. Bell, J. L. O'Brien, Tetrahedron Lett. 1965, 1487.

17) J. Ruppert, V. Bastian, R. Appel, Chem. Ber. 108 (1975) 2329.

18) J. Goubeau, H. W. Wittmeier, Z. Anorg. Allg. Chem. 270 (1952) 16.

19) Weitere Einzelheiten zur Kristallstrukturbestimmung können beim Fachinformationszentrum Karlsruhe, Gesellschaft für wissenschaftlich-technische Information $\mathrm{mbH}, \mathrm{D}-7514$ EggensteinLeopoldshafen 2, unter Angabe der Hinterlegungsnummer CSD54587, der Autoren und des Zeitschriftenzitats angefordert werden.

20) W. Clegg, Acta Crystallogr., Sect. A, 37 (1981) 913.

${ }^{21)}$ G. M. Sheldrick, Univ. Göttingen 1986. 